
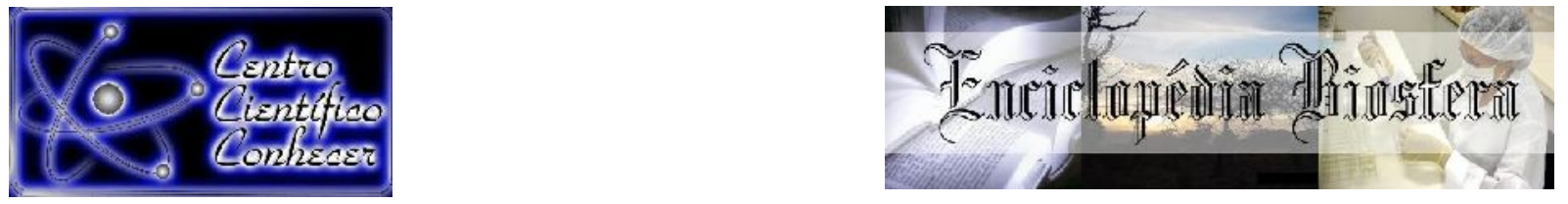

\title{
INVENTÁRIO E ANÁLISE DOS MACROINVERTEBRADOS AQUÁTICOS BIOINDICADORES DA QUALIDADE DA ÁGUA NO RIO URAIM, PARAGOMINAS, PARÁ, BRASIL
}

\author{
Carlos Elias de Souza Braga ${ }^{1}$, Ana Lúcia Nunes Gutjahr ${ }^{2}$ \\ 1. Prof. Dr. Centro de Ciências Sociais e Educação, Universidade do Estado do Pará \\ (bragaelias@yahoo.com.br). Belém, Pará, Brasil. \\ 2. Profa. Dra. Centro de Ciências Sociais e Educação, Universidade do Estado do Pará. \\ Belém, Pará, Brasil.
}

Recebido em: 22/09/2018 - Aprovado em: 23/11/2018 - Publicado em: 03/12/2018 DOI: 10.18677/EnciBio_2018B87

\begin{abstract}
RESUMO
O município de Paragominas, Pará, está localizado na mesorregião Sudeste Paraense e apresenta rios importantes no contexto regional e local, destacando-se os rios Gurupi, Capim, Uraim, Croantá, Piriá e Surubiju, o que torna a região relevante para estudos de monitoramento ambiental nos meios aquáticos e terrestres. Estudos indicam que os macroinvertebrados aquáticos são importantes bioindicadores para o monitoramento de rios, sendo por isso, considerados na avaliação da qualidade hídrica. Este estudo identificou os macroinvertebrados aquáticos em quatro pontos do Rio Uraim, utilizando rede aquática (puçá), para diagnosticar o estado de preservação do mesmo. Foram coletados 211 espécimes de macroinvertebrados aquáticos, pertencentes aos seguintes táxons: Insecta: Odonatas $(n=97)$, Diptera $(n=57)$, Hemiptera $(n=21)$, Coleoptera $(n$ $=11)$ e Ephemeroptera $(n=3)$; Annelida: Oligochaeta $(n=18)$ e Nemathelminthes $(n=$ 4). Em todos os pontos de amostragem houve a dominância de macroinvertebrados classificados como tolerantes, o que indica perturbação ambiental no Rio Uraim, devido à ação antrópica proveniente da cidade de Paragominas, que em toda sua extensão é banhada por esse rio. Este trabalho constitui o primeiro estudo relacionado ao monitoramento ambiental do meio aquático, com o uso de macroinvertebrados no município de Paragominas, o qual poderá servir de base para futuros estudos mais aprofundados relacionados ao assunto.
\end{abstract}

PALAVRAS-CHAVE: Biomonitoriamento, Impacto Ambiental, Poluição hídrica.

\section{INVENTORY AND ANALYSIS OF MACROINVERTEBRATES AQUATIC BIOINDICATORS OF WATER QUALITY OF URAIM RIVER, PARAGOMINAS, PARÁ, BRAZIL}

\begin{abstract}
The municipality of Paragominas, Pará, is located in the southeast region of Pará, and presents important rivers in the regional and local context, especially the Gurupi, Capim,
\end{abstract}


Uraim, Croantá, Piriá and Surubiju rivers, which makes the region relevant for environmental monitoring studies in aquatic and terrestrial environments. Studies indicate that aquatic macroinvertebrates are important bioindicators for the monitoring of rivers and are therefore considered in the evaluation of water quality. This study identified the aquatic macroinvertebrates at four points of the Uraim River, using an aquatic network (puçá), to diagnose the state of preservation of the same. A total of 211 specimens of aquatic macroinvertebrates belonging to the following taxa were collected: Insecta: Odonatas $(n=97)$, Diptera $(n=57)$, Hemiptera $(n=21)$, Coleoptera $(n=11)$ e Ephemeroptera $(n=3)$; Annelida: Oligochaeta $(n=18)$ and Nemathelminthes $(n=4)$. In all the sampling points there was the dominance of macroinvertebrates classified as tolerant, which indicates environmental disturbance in the Uraim River, due to the anthropic action coming from the city of Paragominas, which in all its extension is bathed by this river. This work belongs to the first study related to the environmental monitoring of the aquatic environment, with the use of macroinvertebrates in the municipality of Paragominas, which may serve as a basis for further studies related to the subject.

KEYWORDS: Biomonitoring, Ambiental impact, Water pollution.

\section{INTRODUÇÃO}

As ações humanas têm alterado de forma alarmante os ecossistemas naturais de nosso Planeta, principalmente os ambientes aquáticos, em decorrência de atividades de mineração, construção de represas ou barragens, canalização de cursos de rios, além de processos como a eutrofização (CALLISTO et al., 2001). Tais ações antrópicas mudam, consideravelmente, as características ambientais dos ecossistemas aquáticos, atingindo de forma direta as comunidades biológicas (DUDGEON 1996), que acabam se desestruturando devido às alterações físico-químicas e da qualidade da água do meio aquático do qual dependem para sua sobrevivência (CALLISTO et al., 2001). Quanto à qualidade dos ecossistemas, destacam-se três fatores que podem indicar o nível de "stress" ambiental: 1) a identificação de fatores de risco como resíduos industriais e esgoto; 2) a falta de "stress" definida pelas características físicas e químicas ou indicadores biológicos e 3) a habilidade de um ecossistema em lidar com o "stress" (HOLLING, 1973).

Diante deste cenário de constante degradação ambiental, pesquisas de biomonitoramento vêm demonstrando que organismos aquáticos podem ser utilizados para avaliação da qualidade da água em ecossistemas aquáticos. O biomonitoramento é definido como a utilização sistemática das respostas de organismos vivos para avaliar a degradação ambiental (COSTA et al., 2015). Estudos indicam que os macroinvertebrados aquáticos são importantes bioindicadores para o monitoramento de rios, sendo estes considerados promissoras ferramentas para avaliação da qualidade hídrica (FLOR; SOUTO, 2016; PIMENTA et al., 2016). Diferentes macroinvertebrados apresentam distintas relações à poluição, alguns são característicos de ambientes com águas limpas, tais como os insetos das ordens Plecoptera, Ephemeroptera e Trichoptera, assim como outros que resistem a ambientes pluídos, entre os quais se destacam os dípteros da família Chironomidae e os oligoquetos da família Tubificidae (CALLISTO et al., 2001). Estes animais formam um grupo que indica integralmente os efeitos produzidos por todas as substâncias existentes na água (JUNQUEIRA et al., 2000; REMOR et al., 2013; SCHWAB, 2017). A vantagem de se utilizá-los como 
ferramenta biológica, deve-se a capacidade dos mesmos em detectar os efeitos da perturbação ocorrida há várias semanas ou mais, oferecendo uma visão da situação antes da coleta de amostras (WEIGEL et al., 2002; CARDOSO; NOVAES, 2013; CORTÉS-GUZMÁN; OSPINA-TORRES, 2014).

O município de Paragominas está localizado na mesorregião Sudeste Paraense e possui uma área de $19.309 \mathrm{Km}^{2}$. Atualmente sua paisagem é dominada por ambientes antropizados e matas secundárias (capoeira), com alguns fragmentos de Floresta Ombrófila Densa (IBGE, 2015). O município apresenta rios importantes no contexto regional e local, destacando-se os rios Gurupi, Capim, Uraim, Croantá, Piriá e Surubiju, o que torna a região importante para estudos de monitoramento ambiental nos meios aquáticos e terrestres. Pelo exposto, esse estudo teve como objetivo identificar os macroinvertebrados aquáticos em quatro pontos de coleta no Rio Uraim, no município de Paragominas e diagnosticar os estado de preservação dos ambientes aquáticos às margens do mesmo.

\section{Área de Estudo}

\section{MATERIAL E MÉTODOS} (Figura 1).

O estudo foi realizado no Rio Uraim, município de Paragominas, estado do Pará

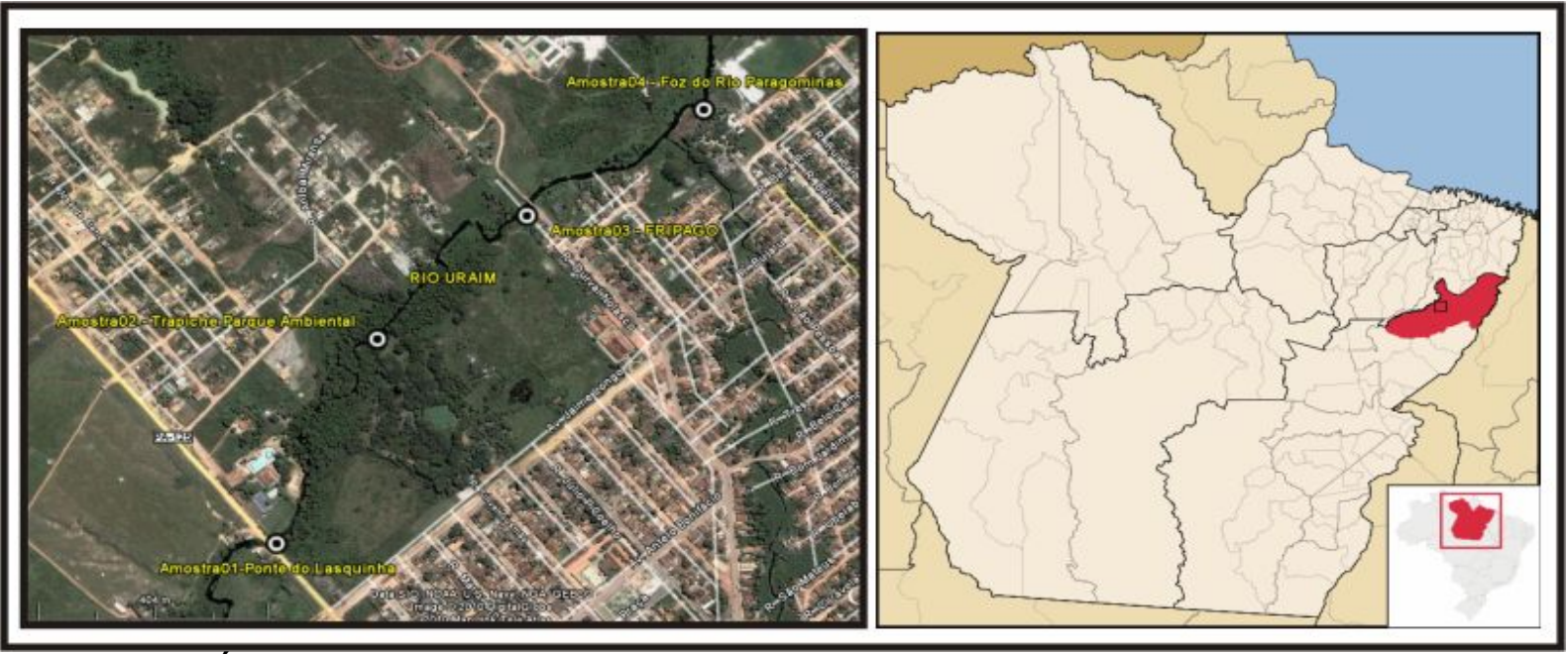

FIGURA 1. Área de estudo na cidade de Paragominas, destacando os pontos de amostragem na margem direita do Rio Uraim. Fonte: Os autores.

\section{Metodologia}

Durante o mês de setembro de 2010 , foram realizadas coletas em quatro pontos de amostragem na margem direita do Rio Uraim (Ponte do Lasquinha, Trapiche Parque Ambiental, FRIPAGO e Foz do Rio Paragominas) na área urbana de Paragominas (Figura 1). 


\section{- Inventário dos macroinvertebrados aquáticos}

A amostragem foi feita por um coletor com auxílio de uma rede de coleta aquática (puçá) com malha de $0,5 \mathrm{~mm}$. As coletas foram padronizadas em cada ponto de amostragem, onde o coletor durante 15 minutos realizava uma varredura com o puçá nas porções inferior e superior da vegetação marginal do Rio Uraim. Todos os pontos de amostragem foram selecionados na margem direita do Rio, devido a maior facilidade de acesso. Após esse procedimento o material coletado foi acondicionado em saco plástico de 50 litros, contendo água do local para que os espécimes chegassem vivos ao laboratório, o transporte de um ponto de coleta até o laboratório levava em média 15 minutos.

O material coletado foi tratado, triado e identificado no laboratório do Campus VI da Universidade do Estado do Pará, essas etapas iniciavam-se em seguida a chegada do mesmo ao laboratório e foram realizadas com o auxílio de um estereomicroscópio (Leica TL 3000) para observação dos espécimes, pinças entomológicas para manipulação dos exemplares, peneiras e bandejas plásticas para separação das amostras e por fim, o material era posto em frascos de vidro com álcool $70 \%$ de acordo com o grupo taxonômico e ponto de coleta. Na identificação do material foram utilizadas chaves dicotômicas de identificação para os macroinvertebrados aquáticos segundo Mugnai et al. (2009).

\section{- Análise da Qualidade Ambiental}

A qualidade ambiental nos pontos de amostragem levantados, considerando os grupos funcionais dos animais coletados, foi classificada em: ECOSSISTEMA NATURAL (presença de macroinvertebrados tolerantes, resistentes com domínio de sensíveis ou intolerantes), ALTERADO (ausência de sensíveis e domínio de tolerantes) e IMPACTADO (presença apenas de resistentes) (GOULART; CALLISTO, 2003).

\section{RESULTADOS E DISCUSSÃO}

Durante o período de amostragem foram coletados 211 espécimes de macroinvertebrados aquáticos, pertencentes aos seguintes grupos: (A) insetos: Odonatas $(n=97)$, Diptera $(n=57)$, Hemiptera $(n=21)$, Coleoptera $(n=11)$ e Ephemeroptera $(n=3) ;(B)$ anelídeos: Oligochaeta $(n=18)$ e (C) vermes: Nemathelminthes $(n=4)$ (Figura 2). As ordens Diptera e Odonata foram encontrados em todos os pontos de amostragem, sendo Diptera mais abundante no ponto denominado Ponte do Lasquinha e Odonata mais abundante na Foz do Rio Paragominas (Figura 2). Ressalta-se que a grande frequência de dípteros nos pontos de amostragem é devido a esses insetos apresentarem distribuição em habitats variados e em diferentes profundidades nos corpos d'água, já em realção as Odonatas é explicado por estas serem na fase jovem (ninfas) predadoras de organismos aquáticos (CALLISTO et al., 2001). Além disso, estudos de Souza et al. (2014) no Alto Rio Paraná, indicaram que em comunidades de macroinvertebrados bentônicos há a prevalência de espécimes das ordens Odonata e Diptera, corroborando com o observado nos pontos de amostragem do Rio Uraim. 


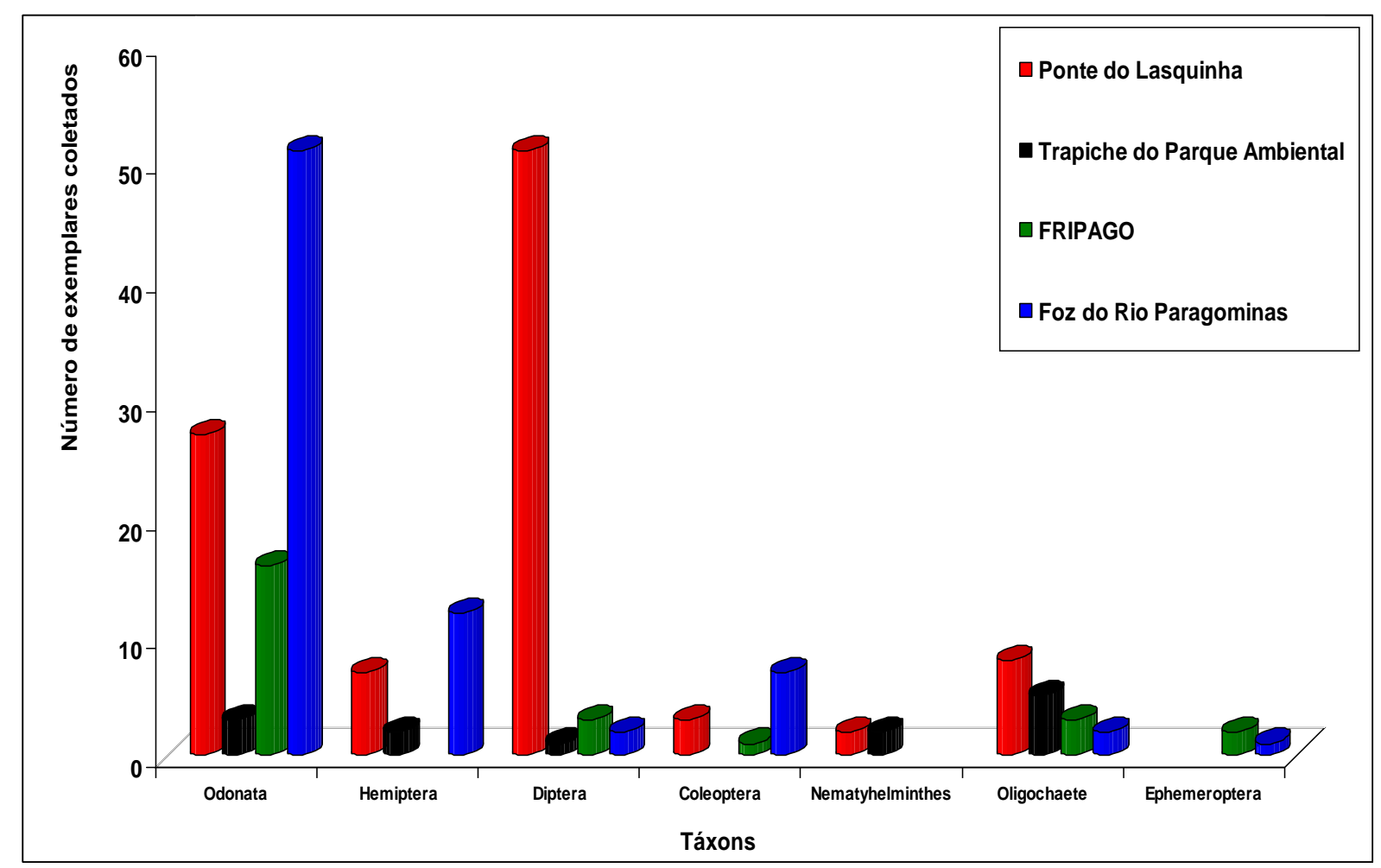

FIGURA 2. Ocorrência de macroinvertebrados por ponto de coleta nas margens do Rio Uraim, Paragominas. Fonte: Os autores.

Durante a realização do estudo, constatou-se que foram encontrados os três grupos de marcroinvertebrados bioindicadores de qualidade ambiental no Rio Uraim, segundo a nomenclatura de Goulart; Callisto (2003), os quais classificam os macroinvertebrados aquáticos em sensíveis (Plecoptera, Ephemerotera e Trichoptera), tolerantes (Coleoptera, Hemiptera, Odonata e Megaloptera) e resistentes (Insecta: Diptera, Mollusca e Anellida).

Também, verificou-se que em todos os pontos de amostragem houve a prevalência dos grupos de macroinvertebrados classificados como tolerantes (Odonata, Hemiptera e Coleoptera) e resistentes (Oligochaeta e Diptera), os quais necessitam de menores concentrações de oxigênio dissolvido na água, representados respectivamente por $61,1 \%$ e $37,4 \%$ dos espécimes coletados, indicando alterações nesses ambientes. Além de 1,4\% macroinvertebrados sensíveis que foram encontrados apenas na Foz do Rio Paragominas e no FRIPAGO (Figura 3). Estudos de Piedras et al. (2006), corroboram os dados observados neste estudo, uma vez que a grande presença de Oligochaeta e Diptera, principalmente da família Chironomidae, indicam haver elevados teores de matéria orgânica e poluentes na água, em ambientes aquáticos. Vale ressaltar que segundo Bubinas; Jaminiené (2001), os Chironomidae e os Oligochaeta são considerados os macroinvertebrados aquáticos mais tolerantes aos poluentes em ambientes bentônicos. 


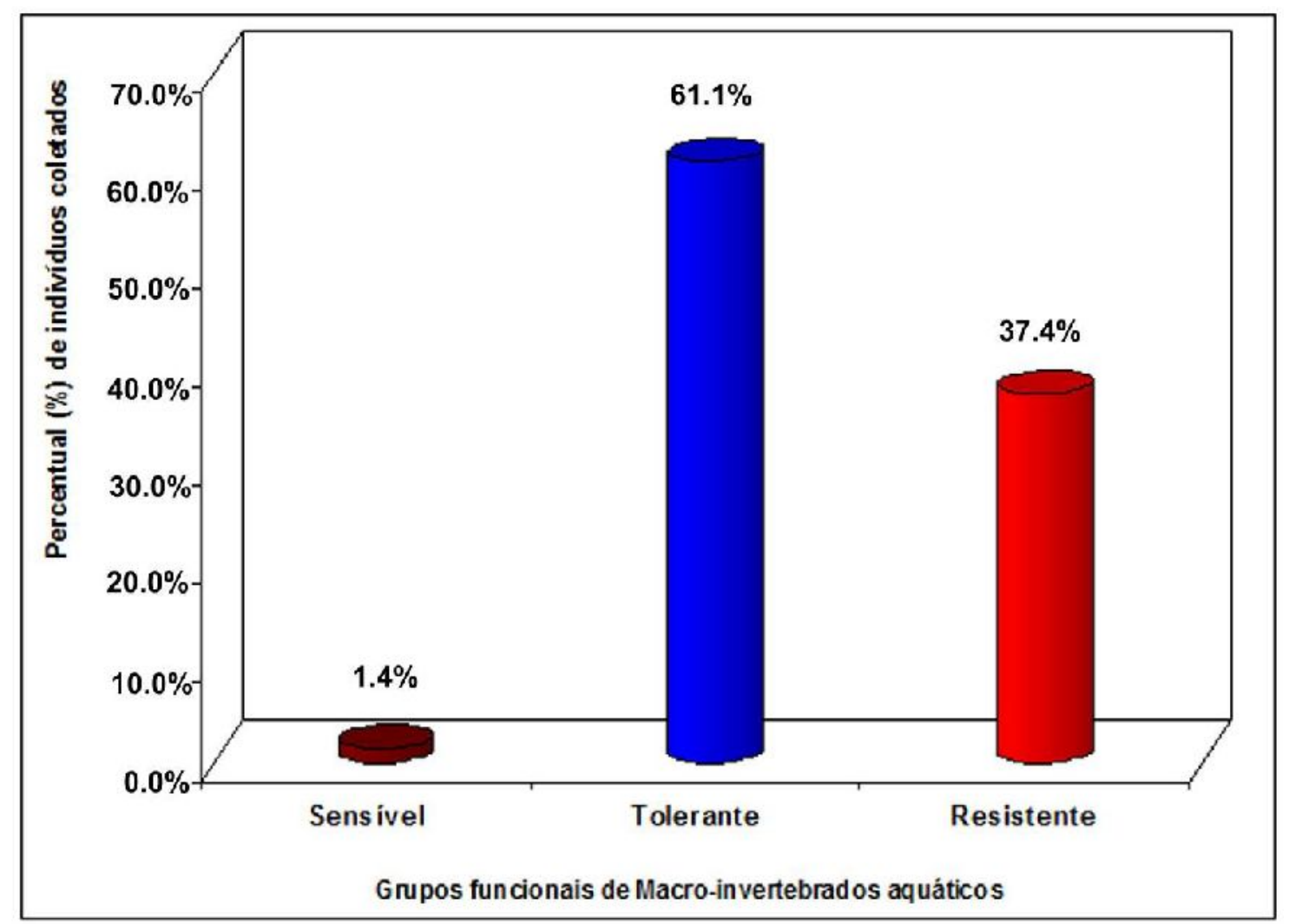

FIGURA 3. Percentual de macroinvertebrados por grupo funcional, coletados no Rio Uraim, Paragominas, Pará. Fonte: Os autores.

Quanto aos pontos de amostragem, destaca-se que a ausência de macroinvertebrados sensíveis nos pontos de coleta localizados na Ponte do Lasquinha e no Trapiche do Parque Ambiental, classifica esses locais como ecossistema Alterado, a ausência desses organismos nesses pontos pode está relacionado ao fato de que no primeiro (Ponte do Lasquinha) encontra-se um baldeário utilizado por banhistas e no segundo (Trapiche do Parque Ambiental) houve a construção de lago artificial no Parque Ambiental, o qual ocasionou a proliferação de algas, que pode ter diminuído a oxigenação da água. Porém, os pontos localizados no FRIPAGO e Foz do Rio Paragominas, por apresentarem, ainda que de forma mínima $(8,0 \%$ e $1,3 \%$, respectivamente) um grupo classificado como sensível (Ordem: Ephemeroptera) e domínio de tolerantes $(68,0 \%$ e $93,3 \%$, respectivamente), classifica esses locais segundo a classificação de Goulart; Callisto (2003), como ecossistema Natural/Alterado (Quadro 1, Figura 2). Portanto, os resultados obtidos deste estudo mostraram que a qualidade da água do Rio Uraim está sofrendo uma notável ação antrópica, a qual atinge de forma diferençada a biota estabelecida deste corpo d'água.

O presente trabalho possui relevante importância por constituir o primeiro estudo relacionado ao monitoramento ambiental do meio aquático, com o uso de macroinvertebrados no município de Paragominas, que poderá servir de base para futuros estudos mais aprofundados relacionado ao assunto. 
QUADRO 1. Pontos de coleta, grupos funcionais de macroinvertebrados e diagnóstico do ambiente, no Rio Uraim, município de Paragominas, Estado do Pará, segundo classificação de Goulart; Callisto (2003).

\begin{tabular}{|c|c|c|c|c|}
\hline \multirow{2}{*}{ Ponto de Coleta } & \multicolumn{3}{|c|}{ Grupos de Macroinvertebrados } & \multirow{2}{*}{$\begin{array}{c}\text { Diagnóstico do } \\
\text { Ambiente }\end{array}$} \\
\hline & Sensível & Tolerante & Resistente & \\
\hline Ponte do Lasquinha & Ausente & Presente & Presente & $\begin{array}{c}\text { Ecossistema } \\
\text { Alterado }\end{array}$ \\
\hline $\begin{array}{c}\text { Trapiche do Parque } \\
\text { Ambiental }\end{array}$ & Ausente & Presente & Presente & $\begin{array}{c}\text { Ecossistema } \\
\text { Alterado }\end{array}$ \\
\hline FRIPAGO & Presente & Presente & Presente & $\begin{array}{c}\text { Ecossistema } \\
\text { Natural/Alterado }\end{array}$ \\
\hline $\begin{array}{c}\text { Foz do Rio } \\
\text { Paragominas }\end{array}$ & Presente & Presente & Presente & $\begin{array}{c}\text { Ecossistema } \\
\text { Natural/Alterado }\end{array}$ \\
\hline
\end{tabular}

\section{CONCLUSÃO}

Concluiu-se que nos pontos de amostragem do Rio Uraim houve a maior abundância de macroinvertebrados das ordens Diptera e Odonata e a prevalência de macroinvertebrados considerados tolerantes e resistentes. Também, que os ambientes aquáticos amostrados no Rio Uraim foram considerados ecossistemas alterado e natural/alterado.

\section{REFERÊNCIAS}

BUBINAS, A.; JAGMINIENÉ, I. Bioindication of ecotoxity according to community structure of macrozoobenthic fauna. Acta Zoológica Lituanica, v. 11, n. 1, p. 90-99, 2001.

CALLISTO, M.; MORETTI, M.; GOULART, M. Macroinvertebrados Bentônicos como Ferramentas para Avaliar a Saúde de Riachos. Revista Brasileira de Recursos Hídricos, v. 6 , n. 1, p. 71-82, 2001. Disponível em: http://labs.icb.ufmg.br/benthos/index_arquivos/pdfs_pagina/callisto.et.al.2001.RBRH.pdf $>$

CARDOSO, R. S.; NOVAES, C. P. Variáveis limnológicas e macroinvertebrados bentônicos como bioindicadores de qualidade da água. Revista Nacional de Gerenciamento de Cidades, v. 1, n. 5, p. 16-35, 2013. Disponível em: 
$<$ http://www.amigosdanatureza.org.br/publicacoes/index.php/gerenciamento_de_cidade s/article/view/510/536>

CORTÉS-GUZMÁN, D.; OSPINA-TORRES, R. Comunidades de macroinvertebrados acuáticos en quebradas de la Isla de Providencia, Mar Caribe Colombiano. Revista Intropica, v. $\quad 9, \quad$ p. 9 9-22, 2014. Disponível em: <http://revistas.unimagdalena.edu.co/index.php/intropica/article/view/1421>

COSTA, G. M; CASSANEGO, M. B. B.; PETRY, C. T.; SASAMORI, M. H.; ENDRES JÚNIOR, D.; DROSTE, A. Avaliação da influência do tempo de exposição de Tradescantia pallida var. purpurea para biomonitoramento da genotoxicidade do ar atmosférico. Revista Brasileira de Biociências, v. 13, n. 4, p. 224-230, 2015. Disponível em: < http://www.ufrgs.br/seerbio/ojs/index.php/rbb/article/view/3348>

DUDGEON, D. Anthropogenic influences on Hong Kong streams. Geo Jounal, v. 40, n. 1-2, p. 53-61, 1996.

FLOR, T. R.; SOUTO, H. N. Biomonitoramento do lago poço verde situado na região de coromandel (MG), utilizando macroinvertebrados bentônicos como indicadores de qualidade da água. Getec, v.5, n.10, p.12-30, 2016. Disponível em: < http://www.fucamp.edu.br/editora/index.php/getec/article/view/795>

GOULART, M. ; CALLISTO, M. Bioindicadores de qualidade da água como ferramenta em estudos de impacto ambiental. Revista da FAPAM, v. 1, n. 1, p. 1-9, 2003.

HOLLING, C. S. Resilience and stability of ecological systems. Annual Review of Ecology and Systematics, v. 4, p. 1-23, 1973.

IBGE. Mapas interativos. 2015. Disponível em: <http://www.ibge.gov.br/mapas>. Acesso em: 20 de set 2018.

JUNQUEIRA, V. M.; AMARANTE, M. C.; DIAS, C. F. S.; FRANÇA, E. S. Biomonitoramento da qualidade das águas da Bacia do Alto Rio das Velhas (MG/Brasil) através de macroinvertebrados. Acta Limnológica Brasiliensia, v. 12, p. 73-87, 2000.

MUGNAI, R., NESSIMIAN, J. L.; BAPTISTA, D. F. Manual de identificação de macroinvertebrados aquáticos do Rio de Janeiro. $1^{\text {a }}$ ed., Brochura Technical Books, p. 176, 2009.

PIEDRAS, S. R. N.; BAGER, A.; MORAES, P. R. R.; ISOLDI, L. A.; FERREIRA, O. G. L.; HEEMANN, C. Macroinvertebrados bentônicos como indicadores de qualidade de água na Barragem Santa Bárbara, Pelotas, RS, Brasil. Ciência Rural, v. 36, n. 2, p. 494-500, 2006.

PIMENTA, S. M.; BOAVEnTURA, G. R.; PEÑA, A. P; RIBEIRO, T. G. Estudo da qualidade da água por meio de bioindicadores bentônicos em córregos da área rural $\mathrm{e}$ 
urbana. Ambiente \& Água, v. 11, n. 1, p. 198-210, 2016. Disponível em: <http://www.scielo.br/pdf/ambiagua/v11n1/1980-993X-ambiagua-11-01-00198.pdf>. doi:10.4136/1980-993X

REMOR, M. B.; HERMOSO, M.; SGARBI, L. F.; PRESTES, T. M. V.; CÂMARA, C. D. Qualidade da água do Rio das Pedras utilizando macroinvertebrados bentônicos como bioindicadores. Cultivando o Saber, v.6, n. 2, p. 97-111, 2013. Disponível em: <https://www.fag.edu.br/upload/revista/cultivando_o_saber/52017fbdbf591.pdf>

SCHWAB, E. J. Macroinvertebrados bentônicos na avaliação de impactos ambientais. Caderno Meio Ambiente e Sustentabilidade, v. 11, n. 6, p. 100-117, 2017. Disponível em:

<https://www.uninter.com/cadernosuninter/index.php/meioAmbiente/article/view/483/49>

SOUZA, F.; SANTOS, C. J.; TRAMONTE, R. P.; KLEPKA, V. Estrutura da comunidade de macroinvertebrados em três córregos na bacia do Alto Rio Paraná: uma relação entre qualidade ambiental e parâmetros ecológicos. Revista em Agronegócios e Meio Ambiente, v. 7, n. 2, p. 413-427, 2014. Disponível em: <https://www.researchgate.net/profile/Fagner_Souza2/publication/286601664_The_stru cture_of_macroinvertebrate_community_in_three_streams_of the_high_river_Parana_b asin_Brazil_Relationship_between_environmental_quality_and_ecological_parameters/li nks/568aab6d08ae1e63f1fbe26c.pdf>

WEIGEL, B. M.; HENNE, L. J.; MARTÍNEZ-RIVERA, L. M. Macroinvertebrate - based index of biotic integrity for protection of streams in west-central Mexico. Journal of the North American Benthological Society, v. 21, n. 4, p. 686-700, 2002. 